\section{YOUNG PEOPLE WITH HYPERTENSION HAVE CEREBRAL VASCULAR ABNORMALITIES THAT COULD IMPAIR CEREBRAL AUTOREGULATION SUPPORTING THE SELFISH BRAIN HYPOTHESIS}

${ }^{1}$ Thomas Hinton*, ${ }^{2}$ Panduka Jayawardena, ${ }^{1} J o n a t h a n$ Rodrigrues, ${ }^{1}$ Nathan Manghat, ${ }^{2}$ Julian Paton, ${ }^{1}$ Angus Nightingale. ${ }^{1}$ Bristol Heart Institute; ${ }^{2}$ University of Bristol

10.1136/heartjnl-2017-311726.86

Background High blood pressure (BP) in childhood is the strongest predictor of hypertension later in life suggesting that the mechanisms responsible are apparent early in life.

The selfish brain hypothesis postulates that impaired cerebral auto-regulation in susceptible individuals results in relative hypoperfusion of the brain-stem. The brain responds by activating the sympathetic nervous system to raise BP. Cerebral vascular anomalies such as vertebral artery hypoplasia or incomplete Circle of Willis would make the brain-stem more vulnerable to this hypo-perfusion. It is unclear whether these vascular anomalies are the cause of the hypertension or a consequence of vascular re-modelling due to hypertension.

We speculated that if these cerebral vascular anomalies were causative, they should have a high prevalence in young onset hypertensives. Conversely, if these cerebral vascular anomalies were secondary to hypertension, we would expect a low prevalence of cerebral arterial remodelling in a group of people with mild hypertension and a short duration of high BP.

Methods We undertook a retrospective analysis of all patients under the age of 40 years referred to our Hypertension Clinic between November 2011 and June 2016. As recommended by NICE, we looked for secondary causes and evidence of end organ damage in this population. Our routine protocol for young onset hypertension is to perform biochemical and anatomic screening (using MRI) for secondary causes of hypertension. We collected MRI data on cerebral vascular anomalies in addition to imaging the heart, aorta, adrenals and kidneys.

Results 65 patients, mean age 30 years $(42 \mathrm{M}, 23 \mathrm{~F})$ were included. Mean clinic BP was $147 / 90 \mathrm{mmHg}$. 62 underwent comprehensive MRI scanning. A clear anatomical cause for the hypertension was found in 2 people $(1$ coarctation and 1 hypoplastic kidney). LVH was seen in 15 (24\%).

Interestingly, cerebral artery anatomical variants (vertebral artery hypoplasia and/or incomplete Circle of Willis) were detected in 26 people (42\%).

Discussion/Implications Cerebral vascular anomalies were common in this population of young people with mild hypertension and only a short duration of high blood pressure. This supports the concept that brain-stem hypo-perfusion might have a pathophysiological role in the aetiology hypertension. This would have important consequences highlighting the need to investigate possible causes of cerebral vascular anomalies in utero or during childhood. Longitudinal studies will help establish whether this causative role is plausible.

The selfish brain hypothesis also suggests that a low target BP may not be appropriate in some people where the initial cause of the hypertension is sympathetic activation due to brain-stem hypo-perfusion. It might explain the "foggy brain" that many hypertensive patients describe when their BP drops after medication.

\section{THE HAEMODYNAMIC EFFECTS OF AN ILIAC ARTERIOVENOUS FISTULA TO TREAT HYPERTENSION ASSESSED USING CPET AND ECHO PARAMETERS}

${ }^{1}$ William Eysenck*, ${ }^{1}$ Jet vanZalen, ${ }^{2}$ Guy Lloyd, ${ }^{1}$ Andrew Marshall, ${ }^{1}$ Ragu Shunmugam, ${ }^{1}$ Steve Furniss, ${ }^{1}$ Neil Sulke. ${ }^{1}$ Cardiology Research Department, Eastbourne Hospital, East Sussex Healthcare NHS Trust; ${ }^{2}$ St Bartholomew's Hospital, Barts Heart Centre

\subsection{6/heartjnl-2017-311726.87}

Introduction A central stent derived arterio-venous iliac fistula using the ROX Coupler reduces blood pressure (BP) in patients (pts) with resistant and/or uncontrolled hypertension (HTN). We performed detailed stress echocardiograms (echos) during concurrent cardiopulmonary exercise testing (CPET) pre and post insertion of a ROX Coupler for HTN. We evaluated the pre and post ROX Coupler characteristics of pts who might progress to cardiac decompensation.

Methods 8 pts aged 46-78 (75\% male) underwent CPET echos at baseline and between 1 and 6 months following ROX Coupler insertion. 1 pt had evidence of late decompensation with persistent ankle swelling not associated with venous stenosis and IVC $>2.5 \mathrm{~cm}$

Results Interim analysis suggests there were no significant differences for any CPET parameter before and after coupler insertion. However TR Vmax, a surrogate for pulmonary artery systolic pressure, significantly increased from 1.96 $\pm 0.94 \mathrm{~m} / \mathrm{s}$ to $2.08 \pm 0.94 \mathrm{~m} / \mathrm{s}, \mathrm{p}=0.01$. Meanwhile, lateral $\mathrm{E} / \mathrm{E}$, a marker of left ventricular filling pressure, significantly decreased from $12.7 \pm 7.2$ to $10.7 \pm 3.8, \mathrm{p}=0.005$.

Conclusions A lower E/E lateral post ROX suggests the left ventricular filling pressure is reduced and the heart is working at a more efficient match of preload and afterload resulting in reduced cardiac oxygen consumption and reduced myocyte stress. An ongoing study is assessing the effect of the ROX Coupler upon AF outcomes by reversing the structural and electrical effects induced by longstanding HTN. These findings may support this hypothesis.

\section{DEVELOPMENT AND EXTERNAL VALIDATION OF A MULTIVARIABLE MODEL OF PRE-TEST LIKELIHOOD OF CORONARY ARTERY DISEASE BASED ON A CONTEMPORARY UK POPULATION, WITH COMPARISON TO EXISTING RISK MODELS}

${ }^{1}$ James RJ Foley* ${ }^{2}$ Colin C Everett, ${ }^{3}$ David Cairns, ${ }^{1}$ Petra Büsterveld, ${ }^{1}$ David P Ripley, ${ }^{1}$ Sven Plein, ${ }^{3}$ Linda Sharples, ${ }^{3} J u l i a$ Brown, ${ }^{1} J o h n$ P Greenwood. 'University of Leeds ${ }^{2}$ Clinical Trials Research Unit, University of Leeds; ${ }^{3}$ Leeds Institute of Clinical Trials Research, University of Leeds

\subsection{6/heartjnl-2017-311726.88}

Introduction Pre-test likelihood models recommended in current UK and US guidelines have been shown to overestimate the probability of coronary artery disease(CAD). We aimed to develop a UK population-based multivariable risk model from CE-MARC(a contemporary study of stable CAD where all patients underwent $\mathrm{X}$-ray coronary angiography) and validate it prospectively in the CE-MARC 2 trial population.

Methods CE-MARC (development population) enrolled patients between 2006-9 with suspected angina. Multivariable logistic regression modelled presence of significant stenosis 